\title{
STANHOPEINAE MESOAMERICANAE II (ORCHIDACEAE). DOS ESPECIES NUEVAS: POLYCYCNIS BLANCOI Y CORYANTHES MADUROANA
}

\author{
GÜNTER GERLACH \\ Botanischer Garten München-Nymphenburg \\ Menzinger Str. 65, 80638 München, Germany. gerlach@botanik.biologie.uni-muenchen.de
}

\begin{abstract}
Two new species of Stanhopeinae (Orchidaceae) are described and illustrated in this paper: Polycycnis blancoi, from Costa Rica, and Coryanthes maduroana from Panama.

RESUMEN. Se describen e ilustran dos especies nuevas de Stanhopeinae (Orchidaceae): Polycycnis blancoi, de Costa Rica, y Coryanthes maduroana de Panamá.
\end{abstract}

Palabras Clave / Key words: Orchidaceae, Stanhopeinae, Coryanthes, Polycycnis, new orchid species, Mesoamérica.

Polycycnis blancoi G. Gerlach, spec. nova

TIPO: Costa RicA. Fila Costeña a lo largo de la división territorial entre las provincias San José y Puntarenas: Refugio de Vida Silvestre Boracayán, aproximadamente $10 \mathrm{~km}$ al este de Dominical, cerca de la Catarata de San Luis, a lo largo de la Fila Tinamastes, $09^{\circ} 14^{\prime} 57,89^{\prime \prime} \mathrm{N}, 83^{\circ} 45^{\prime} 01,48^{\prime \prime} \mathrm{W}, 1008 \mathrm{~m}$, M. Blanco, F. Pupulin, H. León-Páez, G. Gerlach s.n., 30 mayo 2003, floreció en invernadero del Jardín Botánico de Múnich-Nymphenburg, Alemania, octubre 2003, bajo el número 03/2628 (Holotipo: USJ-84851).

FIG. 1-3.

Selva siempreverde, densa y muy húmeda. Planta epífita en la base de un árbol, entre musgos, en la cresta de la fila en una parte sumamente húmeda. Junto con plantas como Drymonia macrantha (Gesne-riaceae), Besleria hirsuta (Gesneriaceae), Tovomita weddelliana (Clusiaceae), Pitcairnia brittoniana (Bromeliaceae), Kefersteinia costaricencis (Orchidaceae), Topobea parasitica (Melastomataceae) y Psammisia ulbrichiana (Ericaceae).

P. blancoi inflorescentias pendentes et lobulos lateralibos hypochili acutos possidet ut in speciebus $P$. morganii Dodson, P. trullifera D.E. Benn. \& Christenson, P. silvana F. Barros subgeneris Polycycnis. P. blancoi distincta est ab P. trullifera e Peruvia floribus minoribus (longitudo labelli 1,65 cm versus 3,3 cm), ab P. silvana e Brasilia lobulis lateralibus hypochili brevioribus (ratio longitudo:latitudo in P. blancoi 1:1, in P. silvana 1:2) et ab P. morganii ex Aequatoria inflorescentiis brevioribus et densioribus et ovariis (cum pedunculis) brevioribus ( $P$. blancoi inflorescentia 15 floribus longitudinae $17 \mathrm{~cm}$, ovario cum pedunculo 1,4 cm; P. morganii inflorescentia 15 floribus longitudinae $45 \mathrm{~cm}$, ovario cum pedunculo $3,0 \mathrm{~cm}$ ).

DESCRIPCIÓN: Planta epifítica, pseudobulbos unifoliados, máximo $3,5 \mathrm{~cm}$ de alto y 3,0 $\mathrm{cm}$ de diámetro, levemente aplanados, piriformes, en corte transversal levemente romboides, apicalmente con un anillo distintivo de color marrón; hoja peciolada, pecíolo de 7,5 $\mathrm{cm}$, lámina plicada, 49,0 $\mathrm{cm}$ de largo por $10,0 \mathrm{~cm}$ de ancho, con 7 nervios prominentes; inflorescencia péndula, densa, $17 \mathrm{~cm}$ de largo, con 15 flores, raquis, pedúnculo y ovario hirsutos, de color oliváceo con pelos marrones, raquis revestido con varias vainas tubulosas, hirsutas, ovario más pedicelo $1,4 \mathrm{~cm}$ de largo, brácteas lanceoladas, cóncavas, acuminadas, en la cara externa pubescentes; flores con sépalos y pétalos de color oliváceo pálido, con barras transversales o puntos rojo-marrón, labelo amarillo vivo en el hipoquilo y el ápice del epiquilo con barras transversales o puntos rojo-marrón, columna verde, con alas purpúreas; sépalos y pétalos patentes hasta levemente reflexos, sépalos cóncavos, libres, sépalo dorsal lanceolado, acuminado, $2,1 \mathrm{~cm}$ de largo y (cuando aplanado) $0,65 \mathrm{~cm}$ de ancho, sépalos laterales levemente asimétricos, pétalos angostamente oblanceolados, algo 


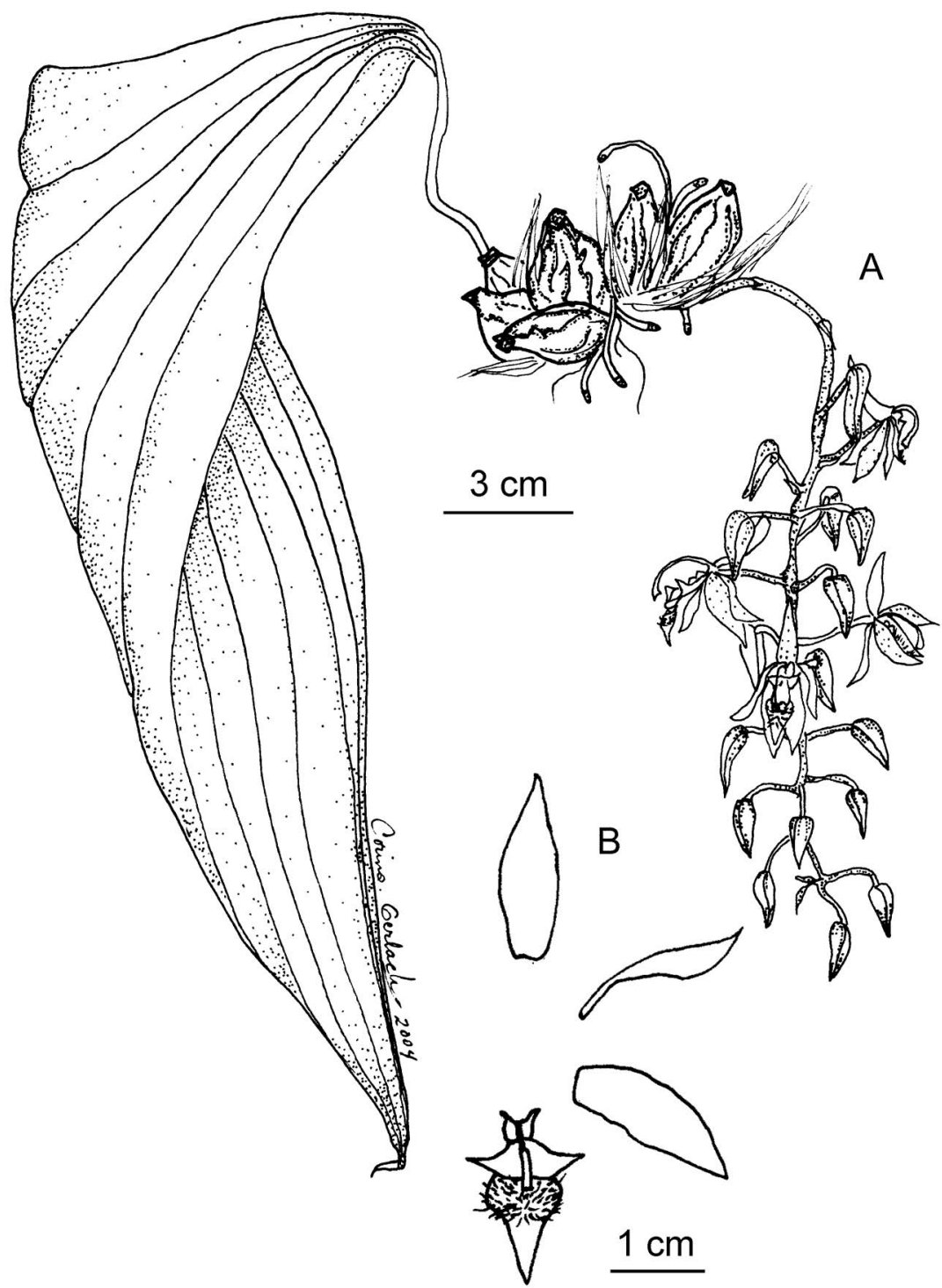

Fig. 1. Polycycnis blancoi G. Gerlach. A - Hábito. B - Sépalos, pétalo y labelo, extendidos. Dibujado del holotipo. Ilustración: Corina Gerlach.

estipitados, sigmoides, $2,0 \mathrm{~cm}$ de largo y $0,35 \mathrm{~cm}$ de ancho; labelo cortamente unguiculado, $1,65 \mathrm{~cm}$ de largo, en la base con dos aurículas erectas, obtusas, 0,2 cm de largo; hipoquilo en el centro con una quilla longitudinal, elevándose más hacia el ápice, sobrepasando el epiquilo y dos lóbulos laterales subtriangulares, agudos, cuando aplanados de extremo a extremo midiendo $1,1 \mathrm{~cm}$, parte central densamente pubescente a lo largo de la quilla; epiquilo truliforme hasta romboide, agudo, $1,1 \mathrm{~cm}$ de largo y $0,8 \mathrm{~cm}$ de ancho, en la base con pelos largos esparcidos; columna arqueada, delgada, clavada en el extremo distal, con alas redondeadas, rostelo tridentado, diente medio muy largo y delgado, agudo, los dientes laterales en parte unidos con el medio por una película hialina, clinandrio casi plano; polinario con un viscidio agudo, un estípite linear y delgado y dos polinios angostamente oblongoides.

DiAgNóSTICO DIFERENCIAL: La nueva especie tiene con las especies $P$. morganii, $P$. trullifera y $P$. silvana 


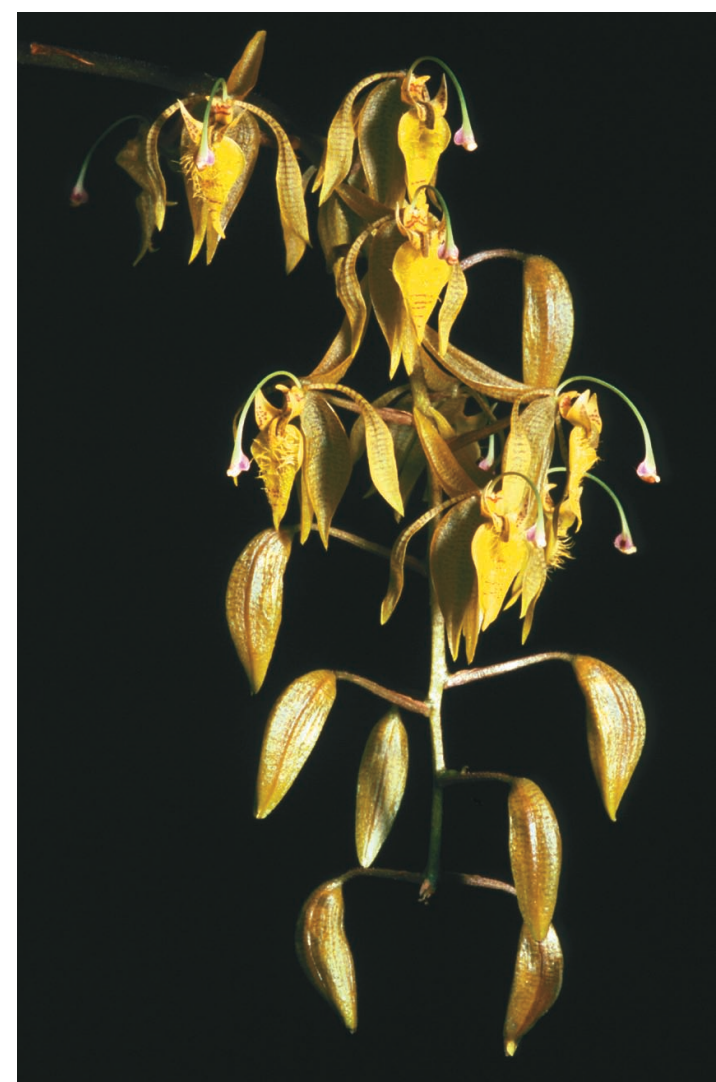

Fig. 2. Polycycnis blancoi G. Gerlach. Inflorescencia. Fotografía del holotipo.

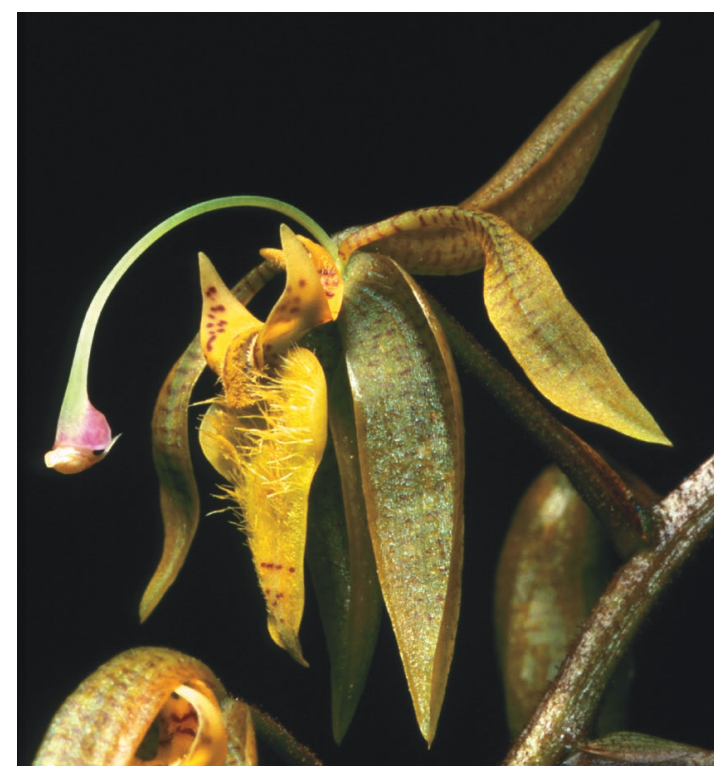

Fig. 3. Polycycnis blancoi G. Gerlach. Flor. Fotografía del holotipo. en común inflorescencias péndulas y lóbulos laterales del hipoquilo agudos. Se diferencia de $P$. trullifera de Brasil por sus flores más pequeñas (largo del labelo $1,65 \mathrm{~cm}$ versus $3,3 \mathrm{~cm}$ ), de $P$. silvana de Brasil por los lóbulos laterales del hipoquilo más anchos (relación largo a ancho de $P$. blancoi $1: 1$, de $P$. silvana 1:2), y de $P$. morganii por sus inflorescencias más cortas y más densas y el ovario más corto $(P$. blancoi - 15 flores, $17 \mathrm{~cm}$ de largo, ovario más pedicelo 1,4 cm; P. morganii - 15 flores, $45 \mathrm{~cm} \mathrm{de}$ largo, ovario más pedicelo $3,0 \mathrm{~cm}$ ).

OBSERVACIONES: Como la única planta conocida no era de las más grandes y se había establecido recientemente en invernadero, es muy probable que en el futuro crecerá y florecerá con una inflorescencia más larga y con más flores.

Hasta ahora existen 17 especies válidas en el género Polycycnis. Éste puede dividirse en dos subgéneros:

1) Subgénero Polycynis - plantas epifíticas o terrestres, inflorescencias erectas o péndulas, epiquilo más o menos truliforme.

1.1) Especies con inflorescencias erectas:

P. escobariana G. Gerlach: Colombia, Ecuador $P$. grayi Dodson: Ecuador

P. muscifera (Lindl. \& Paxt.) Rchb.f. (Cycnoches muscifera Lindl. \& Paxt., Cycnoches buchtienii Krzl., P. acutiloba Schltr.): Venezuela, Colombia, Bolivia

P. pfisteri Senghas, Taggesell \& G. Gerlach: Colombia

P. villegasiana G. Gerlach: Colombia

1.2) Especies con inflorescencias péndulas:

$P$. annectans Dressler: Ecuador

P. blancoi G. Gerlach: Costa Rica (¿también Panamá?)

P. barbata Rchb.f.: Costa Rica, Panamá

P. lehmannii Rolfe: Colombia

P. lepida Linden \& Rchb.f. (sin.: P. gratiosa Endrés \& Rchb.f.): Costa Rica, Panamá, Colombia

P. morganii Dodson: Colombia, Ecuador P. trullifera D.E. Benn. \& Christenson: Perú P. silvana F. Barros: Brasil 
2) Subgénero Angustilabia - plantas epifíticas, inflorescencias siempre péndulas, epiquilo linear.

$P$. aurita Dressler: Colombia

$P$. ornata Garay: Panamá, Colombia, Ecuador

P. surinamensis C. Schweinf.: Surinam, Venezuela

P. tortuosa Dressler: Panamá

Sin duda, la especie nueva $P$. blancoi se puede integrar al subgénero Polycycnis, en el grupo con inflorescencias péndulas. De las especies agrupadas aquí, $P$. barbata y $P$. trullifera tienen las flores más grandes del género. $P$. barbata y $P$. lepida tienen los lóbulos laterales del hipoquilo obtusos y redondeados, mientras $P$. annectans, $P$. blancoi, $P$. lehmannii, $P$. morganii, $P$. silvana y $P$. trullifera $\operatorname{los}$ tienen agudos y triangulares. Polycycnis annectans es una especie muy particular, porque tiene la columna más corta y gruesa que el resto del género; se parece más bien a la columna de una Kegeliella. Los lóbulos laterales del labelo de P. lehmannii son muy angostos y largos, tienen una relación largo por ancho de 3:1 y, así, la especie se distingue fácilmente de todas las otras especies del género. Según estos caracteres exclusivos ya mencionados, quedarían las especies $P$. morganii de Ecuador y P. silvana de Brasil, las más difíciles de distinguir de la nueva especie. Ésta se distingue de $P$. morganii por la inflorescencia más densa y más corta y el ovario con el pedicelo más corto (P. blancoi - 15 flores, $17 \mathrm{~cm}$ de largo, ovario más pedicelo 1,4 cm; P. morganii - 15 flores, $45 \mathrm{~cm}$ de largo, ovario más pedicelo $3,0 \mathrm{~cm}$ ). Polycycnis blancoi se distingue de $P$. silvana por las aurículas un tercio más cortas $(0,2 \mathrm{~cm}$ en vez de $0,3 \mathrm{~cm}$ o más $)$ y la forma de los lóbulos laterales (relación largo:ancho es en $P$. blancoi 1:1, en P. silvana 1:2). Además, existen patrones diferentes de colores del labelo; en $P$. silvana la combinación de rojo con crema es dominante, en $P$. morganii es verde con puntos rojos, mientras que en $P$. blancoi es casi unicolor, amarillo.

Etimología: La especie está dedicada a Mario Blanco, orquideólogo costarricense que encontró la planta investigada durante una excursión de recolecta. Mario se dedica con mucho entusiasmo, desde hace varios años, a la investigación de la orquideoflora de Costa Rica.
Coryanthes maduroana G. Gerlach, spec. nova

TIPO: PANAmÁ: Provincia de Coclé; El Valle de Antón, floreció en cultivo en Panamá, 4 de diciembre 2002, Andrés Maduro y Erick Olmos 512 (Holotipo: PMA).

FIG. 4.

Species nova differt ab speciebus omnibus generis Coryanthis combinatione hypochili minus profundi cum verrucis paucis in mesochilo dorsali

DESCRIPCIÓN: Planta epifítica, cespitosa, siempre creciendo en hormigueros; psedobulbos angostamente oblongoides hasta oblongo-cónicos, profundamente multi-surcados, bifoliados, con un anillo marrón en el ápice, $11,0 \mathrm{~cm}$ de alto y $2,1 \mathrm{~cm}$ de diámetro, cuando jóvenes parcialmente revestidos por vainas membranáceas; hojas angostamente elípticas, hasta linearlanceoladas, agudas, $52 \mathrm{~cm}$ por $4,0 \mathrm{~cm}$, pecíolo indistinto; inflorescencia péndula, $30 \mathrm{~cm}$ de largo, con 3 flores; pedúnculo marrón verdoso, con 4 vainas parduscas, tubulosas, membranáceas, lanceoladas, de 2,3 $\mathrm{cm}$ de largo; brácteas floríferas ovadas, agudas, 3,5 $\mathrm{cm}$ de largo y 1,4 cm de ancho; pedicelo más ovario $9,5 \mathrm{~cm}$ de largo; flores de tamaño medio para el género, de color crema hasta amarillento o verdoso con manchas leopardinas (margen oscuro y centro pálido con una mancha oscura) de un rojo cárneo, pétalos, hipoquilo y mesoquilo solamente con puntos rojo cárneo, uña con manchas alargadas; sépalos fuertemente enrollados; pétalos algo ligulados, falcados, $4,1 \mathrm{~cm}$ de largo y $1,3 \mathrm{~cm}$ de ancho; labelo $7,2 \mathrm{~cm}$ de alto, con la uña de $1,3 \mathrm{~cm}$, ligeramente comprimida dorsalmente; hipoquilo galeiforme, poco profundo, $1,1 \mathrm{~cm}$ de alto y $1,8 \mathrm{~cm}$ de ancho, externamente con un semicírculo piloso, márgenes inflexos, ápice poco emarginado; mesoquilo canaliculado, algo comprimido lateralmente, $2,9 \mathrm{~cm}$ de largo y $1,5 \mathrm{~cm}$ de ancho, con dos líneas de verrugas que siguen a lo largo de su mediana con dos estrías pilosas, su parte visible con dos excrecencias en forma de verrugas cónicas; epiquilo hemisférico, en el ápice tridentado; columna $3,1 \mathrm{~cm}$ de largo, en el tercio distal rectangular, reflexa, en la encorvadura con dos alas redondeadas, en la base con dos pleuridios subcuadrados de $0,6 \mathrm{~cm}$ por $0,7 \mathrm{~cm}$; polinario parecido al de las otras especies. Aroma fuerte y un poco desagradable.

PARATIPO: Panamá. Prov. Veraguas: Santa Fe, leg. I. 

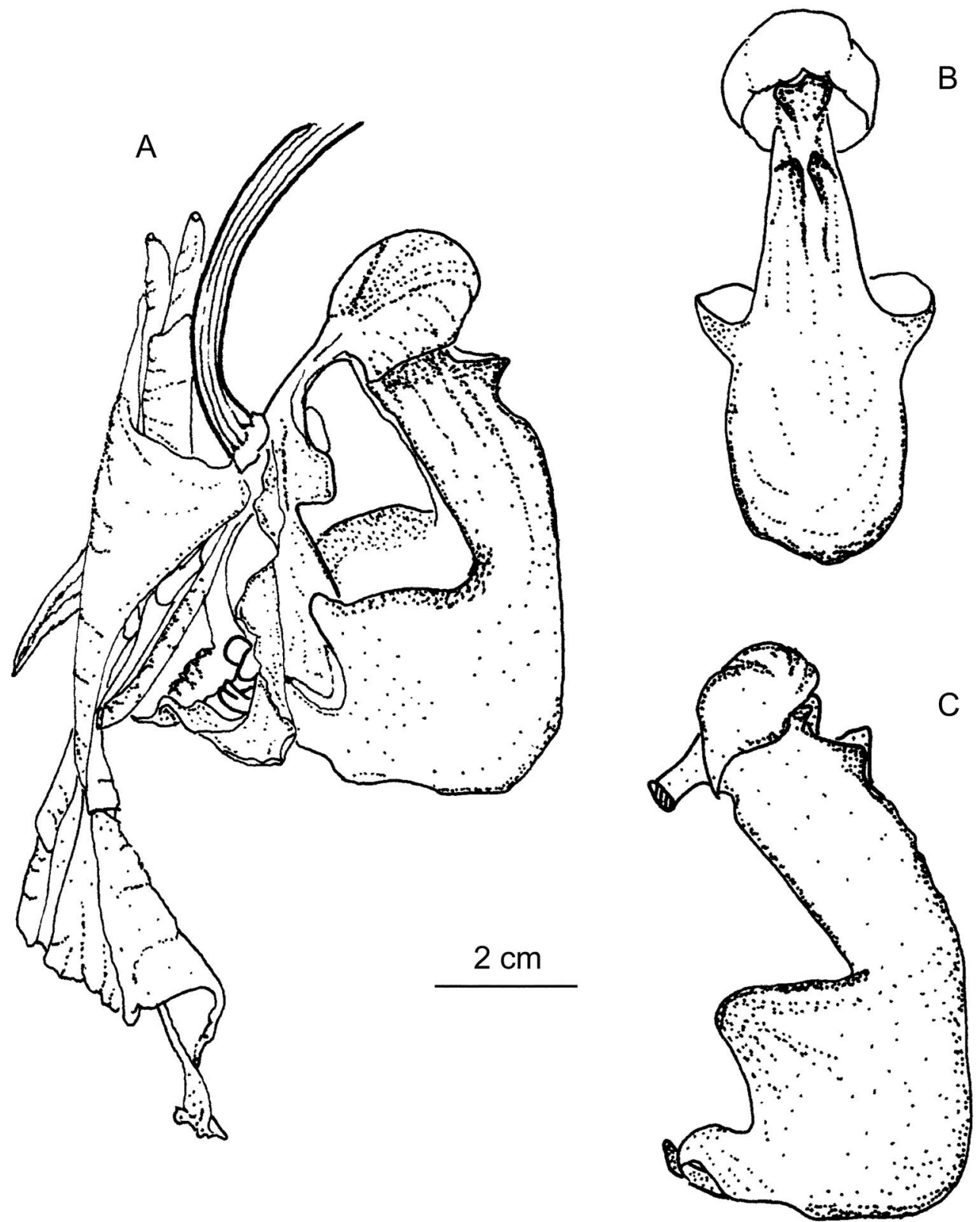

Fig. 4. Coryanthes maduroana G. Gerlach. A - Flor, vista semilateral. B - Labelo, vista dorsal. C - Labelo, vista lateral. Dibujado del paratipo (M). Ilustración: Corina Gerlach.

Zapato s.n. 2002, floreció en cult. en el Jardín Botánico de Múnich, Alemania, 2 de abril 2003, 02/2950 (M).

DIAGNÓSTICO DIFERENCIAL: Se diferencia de todas las otras especies del género Coryanthes por la combinación de un hipoquilo poco profundo con unas pocas verrugas visibles en la base dorsal del mesoquilo.

DISTRIBUCIÓN: Hasta ahora la especie se conoce solamente de Panamá. Así, se trata de una planta endémica en ese país.

ETIMOLOGÍA: La especie está dedicada al Sr. Andrés 
Maduro, quien con sus recolectas y su colección de plantas cultivadas aumentó significativamente el conocimiento de las orquídeas panameñas. Con su inmensa ayuda pude entender mejor la taxonomía del género Coryanthes en Panamá y mejorar muchísimo la identificación de las especies en este país.

OBSERVACIONES: El aroma floral de C. maduroana es notablemente simple; está compuesto solamente por tres moléculas:

$\begin{array}{lr}\begin{array}{l}\text { 2-N-metilaminobenzaldehido } \\ \quad \text { (2-N-methylaminobenzaldehyde) }\end{array} & 94,5 \% \\ \begin{array}{l}\text { metil-N-metilantranilato } \\ \quad \text { (methyl-N-methylanthranilate) }\end{array} & 0,3 \% \\ \begin{array}{l}\text { 2-aminobenzaldehido } \\ \quad \text { (2-aminobenzaldehyde) }\end{array} & 0,2 \%\end{array}$<smiles>Nc1ccccc1C=O</smiles>

\section{2-amino benzaldehyde}<smiles>CNc1ccccc1C=O</smiles>

2-N-methyl aminobenzaldehyde<smiles>CNc1ccccc1C(=O)OC</smiles>

methyl N-methyl anthranilate

Todas estas sustancias pertenecen al mismo camino de biosíntesis. Lo interesante es que el aroma se compone solamente de tres sustancias; normalmente son un mínimo de 10 sustancias ó mas. El aroma de las flores pertenecientes al síndrome floral de perfumes ${ }^{1}$ normalmente no es muy complejo; en unas pocas especies sólo se han encontrado desde uno hasta tres componentes (Gerlach \& Schill 1989, 1991, 1993). El 2-N-metilaminobenzaldehido fue encontrado por primera vez como sustancia natural en el aroma floral de Coryanthes mastersiana Rchb.f. (Gerlach \& Schill 1989). Esta especie se distingue de todas las otras especies del género Coryanthes por la presencia de este compuesto. En cuanto a estructura y color $C$. mastersiana es muy variable, pero como tenemos muchas recolectas de esta especie del Chocó colombiano sabemos bastante sobre su plasticidad en forma y color. Asimismo, sabemos que posee un aroma floral que contiene más de $90 \%$ de $2-\mathrm{N}$-metilaminobenzaldehido. Los primeros intentos de sintetizar 2-N-metilaminobenzaldehido fracasaron, debido a la autopolimerización de la sustancia (así perdió su atractividad) y a problemas con la infraestructura en el Chocó. Finalmente, algunos experimentos con cebos de esta sustancia en la costa del Chocó mostraron en 1994 su atractividad para los polinizadores.

Con las verrugas dorsales visibles sobre el mesoquilo, la especie nueva muestra cierto parentesco con las especies de la sección Lamellunguis, pero el resto de los caracteres (hábito, forma del mesoquilo, aroma floral) la ubican en la sección Coryanthes. Aunque el aroma floral es casi idéntico al de C. mastersiana, se trata sin duda de una especie diferente. Coryanthes mastersiana ha sido recolectada solamente en Colombia, mientras que C. maduroana fue hallada en El Valle de Antón (prov. Coclé) y en Santa Fe (prov. Veraguas), ambos sitios situados al oeste del istmo de Panamá. Además, nunca se han observado flores de C. mastersiana con verrugas en el mesoquilo.

Agradecimientos. Quiero dar las gracias a Franz Schuhwerk (Botanische Staatssammlung München, Alemania) por traducir las descripciones diferenciales al latín, a Corina Gerlach por los dibujos y las correcciones del texto, y a Roman Kaiser (Givaudan Schweiz AG, Dübendorf, Suiza) por los análisis de los aromas florales.

${ }^{1}$ El síndrome floral de perfumes describe un sistema de polinización. Las plantas que exhiben este síndrome son polinizadas únicamente por machos de abejas euglósidas, que buscan y recolectan sustancias aromáticas en las flores. 


\section{Literatura CitADA}

De Barros, F. 1983. Uma nova espécie de Polycycnis (Orchidaceae) do Brasil. Rev. Brasil. Bot. 6: 15-18.

Dodson, C.H. 1980. Polycycnis morganii. Icon. Pl. Trop. 3: 269.

Dressler, R.L. 1977. El género Polycycnis en Panamá y Costa Rica. Orquideología 8(1): 117-127.

Gerlach, G. \& Schill, R. 1989. Fragrance analyses, an aid to taxonomic relationships of the genus Coryanthes (Orchidaceae). Plant. Syst. Evol. 168: 159-165.

Gerlach, G. \& Schill, R. 1991. Composition of Orchid Scents Attracting Euglossine Bees. Bot. Acta 104: 379-391.

Gerlach, G. \& Schill, R. 1993. Die Gattung Coryanthes Hook. (Orchidaceae); eine monographische Bearbeitung unter besonderer Berücksichtigung der Blütenduftstoffe. Trop. Subtrop. Pflanzenwelt 83: 1-205. 